\title{
Management of Bilateral Ectopically Erupting Maxillary Molars: A Case Report
}

\author{
Bourane Ambriss ${ }^{1}$, Carla Moukarzel ${ }^{2}$, Balsam Noueiri ${ }^{3}$
}

\begin{abstract}
Background: Ectopic eruption of the permanent first molar is a common eruption disorder occurring during mixed dentition. The prevalence of this condition ranges from $3 \%$ to $4 \%$ in children. It is described as an abnormal mesioangular eruption path of the first permanent molar with a possibility of initiating the premature resorption of the distal root of the adjacent primary second molar. Non-treatment can result in the early loss of the second primary molar, space loss, and impaction of second premolars. Various treatment modalities have been mentioned in the literature for the management of ectopic eruption.

Aim: This article presents the case of an eight-year-old boy with irreversible bilateral ectopic eruption of the permanent maxillary first molars. Case description: A modifed Croll's appliance was the treatment of choice to correct the path of eruption of the latter molars. An activation of the appliance was performed at intervals of two weeks.
\end{abstract}

Conclusion: The result was obtained six weeks later and a regular follow-up was scheduled.

Keywords: Case report, Distal tipping, Ectopic eruption, Interceptive orthodontic treatment, Permanent maxillary first molars.

International Journal of Clinical Pediatric Dentistry (2019): 10.5005/jp-journals-10005-1602

\section{INTRODUCTION}

In 1923, Chapman was the first author to describe ectopic eruption (EE). ${ }^{1}$ Ectopic eruption of permanent first molars (PFM) occurs due to the molar's abnormal mesioangular eruption path, resulting in an impaction at the distal prominence of the primary second molar crown. ${ }^{2}$ This anomaly can initiate the premature resorption of the distal root of the adjacent primary second molar.,

Young reported a prevalence ranging between $3 \%$ and $4 \%$ of children presenting this condition. ${ }^{5} \mathrm{EE}$ is seen more often in the maxilla rather than the mandible with no difference in prevalence between unilateral and bilateral localization. ${ }^{6,7}$ Regarding the distribution of this anomaly among genders, there is no consensus about which sex is more affected.

The etiology of the EE of PFMs is poorly known, although a multitude of local and systemic factors have been involved. ${ }^{8-10}$ Unusually, large PFMs combined with discrepancies in the arch size and a mesially inclined path of eruption are among the most likely causes of EE. ${ }^{4-10}$

In 1957, Young described two types of EE. $66 \%$ of the cases belong to the reversible type, also called "jump" type, where PFM spontaneously corrects its path of eruption; and the irreversible type, also called the "hold" type, where the tooth remains locked in its ectopic position in the absence of any treatment or until the premature exfoliation of the second primary molar. ${ }^{5}$

After the diagnosis of EE, an observation period of three to six months is essential to differentiate between these two types to avoid unnecessary treatment. ${ }^{4-7}$ Since there is usually no pain associated with this anomaly, it is only accidently discovered upon clinical and radiological examination in the early mixed dentition. ${ }^{9,11-16}$ Early intervention is very important for the development of proper occlusion by preventing the mesial drift of the permanent molar and early loss of the primary second molar, thus preserving the arch circumference. ${ }^{4,9-12}$

Depending on the severity of the impaction of PFMs, a multitude of treatment modalities have been reported. They can be summed

\begin{abstract}
${ }^{1-3}$ Department of Pediatric Dentistry, Faculty of Dentistry, Lebanese University, Beirut, Lebanon

Corresponding Author: Bourane Ambriss, Department of Pediatric Dentistry, Faculty of Dentistry, Lebanese University, Beirut, Lebanon, Phone: +9613632472, e-mail: b-ambriss@hotmail.com

How to cite this article: Ambriss B, Moukarzel C, et al. Management of Bilateral Ectopically Erupting Maxillary Molars: A Case Report. Int J Clin Pediatr Dent 2019;12(2):153-156.

Source of support: Nil

Conflict of interest: None
\end{abstract}

into two major categories: interproximal wedging for minor impaction and distal tipping of the ectopic molar for severe cases. . $^{3,7-12}$

The present case describes irreversible bilateral ectopically positioned permanent maxillary first molars (PMFMs) with grade III resorption according to Barberia-Leache's classification. ${ }^{13} \mathrm{~A}$ modified Croll's appliance was used to perform the distal tipping of the latter molars. ${ }^{14}$

\section{Case Description}

An eight-year-old male came to the Department of Pediatric Dentistry at the Lebanese University for a general check-up. The patient's medical history was noncontributory. Extraoral examination revealed no significant findings. Intraoral examination showed that he had a mixed dentition. The permanent maxillary first molars (PMFMs) were impacted and showed plaque accumulation. The patient's oral hygiene was good and no caries were detected (Fig. 1). Two bitewings confirmed the absence of interproximal caries. Dental panoramic tomography revealed that two-thirds of the PMFMs' roots were complete (Fig. 2). Moreover, they were obstructed by the distal bulge of the right primary maxillary second molars (55) and the left one (65) causing a premature resorption on their distal roots (Fig. 3). Despite severe resorption, teeth 55 and 65 showed no significant mobility. 


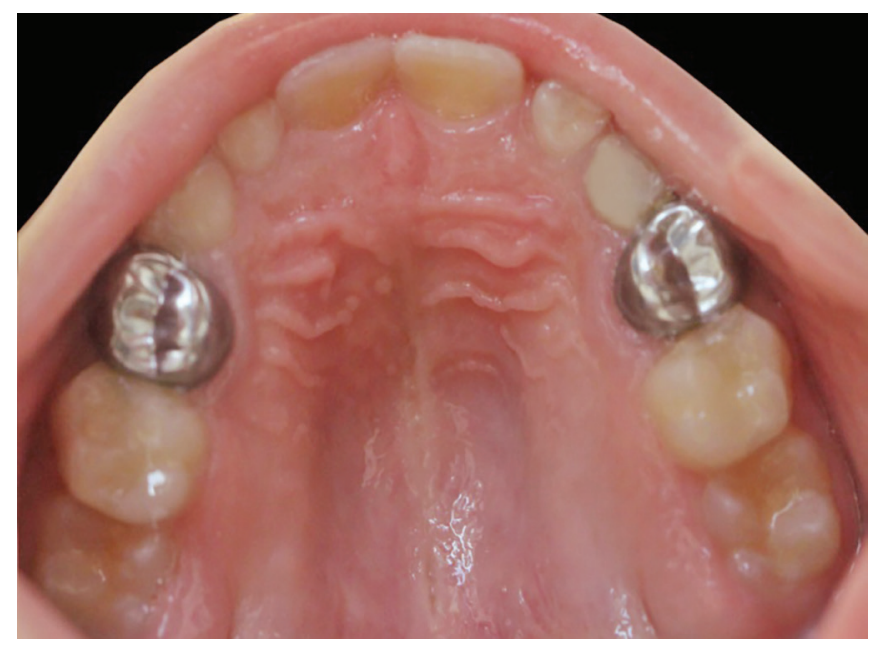

Fig. 1: Preoperative photograph showing partially erupted and mesially inclined permanent maxillary first molars

A modified Croll's appliance was chosen to distalize the PMFMs. It is a fixed appliance with bilateral distal extensions (2-3 mm distal of the right PMFM (16) and the left one (26)). A 0.036 " orthodontic wire was used to fabricate both extensions with small hooks on their distal aspect. A transpalatal bar with an acrylic button is incorporated. Bands are cemented on the first primary molars, while bondable buttons are placed on the mesio-occlusal aspect of teeth 16 and 26 . In order to provide distal pressure on both permanent molars, chain elastics are adapted on both buttons and placed over the hooks on the distal aspect of both extensions (Fig. 4). The activation of the appliance was done every two weeks by shortening the elastic chain. Six weeks later, clinical and radiographic exams revealed that the first molars displayed a favorable position and proper angulation relative to the occlusal plane (Fig. 5). After the correction of EE of PMFMs and the removal of the appliance, the patient was scheduled for follow-up appointments every six months as he was transitioned to a full permanent dentition.

\section{Discussion}

Ectopic eruption of PMFM is a common eruption disorder occurring during mixed dentition. ${ }^{4-15}$

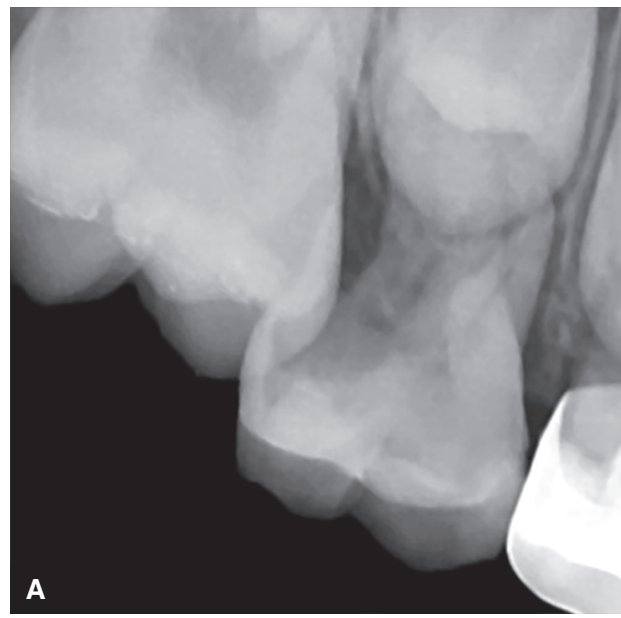

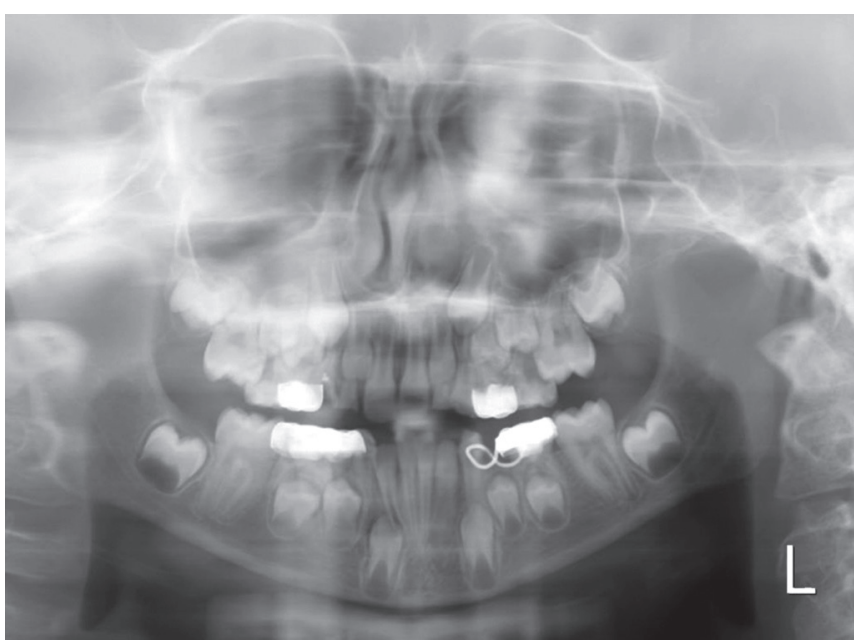

Fig. 2: Panoramic radiograph showing the impaction of tooth (16) and (26)

If left untreated, many problems could arise, such as mesial tipping and rotation of the permanent molar, premature loss of the second primary molar, reduction of the arch length, and impaction of the second premolar. ${ }^{4,9,11-16}$

The case described in this article is about an eight-year-old boy presenting an irreversible bilateral EE of PMFMs with grade III resorption of the primary maxillary second molars. ${ }^{13}$

Many authors agreed that the diagnosis of EE cannot be confirmed unless the patient is at least seven years old, and his/her tooth development was compatible with his/her chronological age. ${ }^{17}$ Although EE sometimes is self-correcting, Bjerklin and Kurol considered that if the first permanent molar has not been fully emerged by age seven, then it is unlikely that natural correction will occur. ${ }^{4-9}$

As for the time of initiating the treatment, Roberts states that correction should be done immediately after the diagnosis is established. ${ }^{18}$ Whereas, Christensen and Fields recommend an observation period of three to six months before any intervention., ${ }^{9,19}$

In this case, the decision to immediately intervene was taken since the patient was eight years old, and the PMFMs reached Nolla developmental stage nine. ${ }^{20}$ Due to the severity of the impaction, distal tipping techniques were considered. ${ }^{16}$

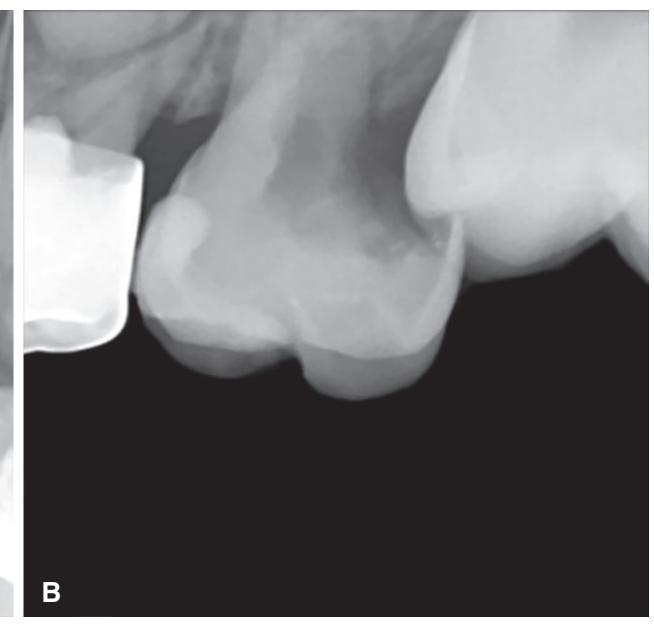

Figs 3 A and B: Preoperative radiographs showing the mesio-occlusal surfaces of PMFMs (16) and (26) impacted under the distal bulge of the second deciduous molars 


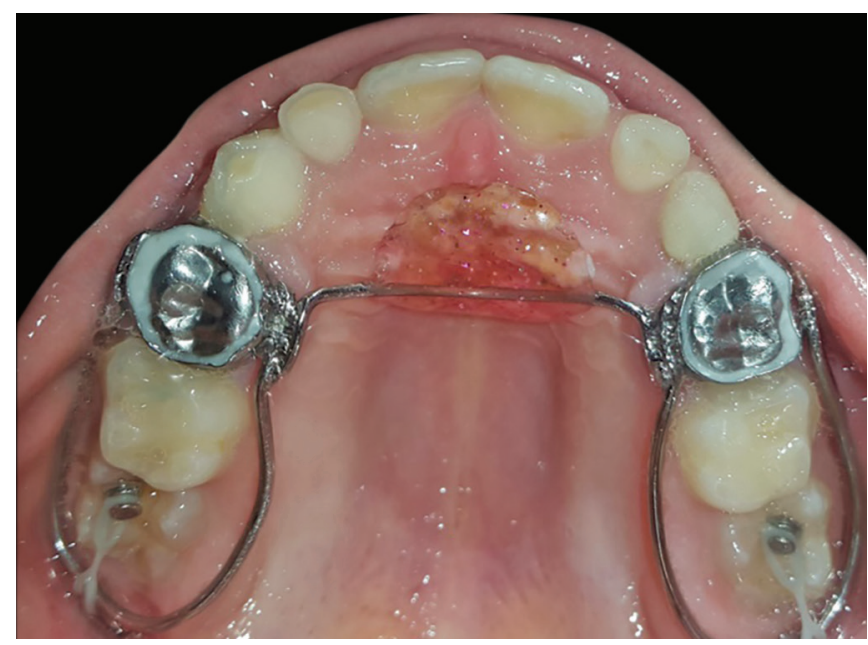

Fig. 4: Cemented modified Croll's appliance

Tipping action can be accomplished using a multitude of fixed and removable appliances (i.e., brass wires, sectional wires with open coil springs, sling shot-type appliances, Halterman appliance, Humphrey appliance, and others). ${ }^{2-16}$

Initially, a Croll's appliance was decided, but since the primary maxillary second molars presented severe root resorption, the right primary first molar (54) and the left one (64) were chosen as an anchor to improve the stability of the appliance and prevent further damage to the primary second molars. ${ }^{14-21}$ Radiological examination showed that 54 and 64 underwent pulpotomy treatment and were properly restored by stainless steel crowns. Upon clinical examination, no symptoms were detected.

The bonded buttons were placed on the distal slope of the mesiobuccal cusps of the first permanent molars as described by Kennedy. ${ }^{22}$ This position helps the reduction of occlusal trauma and provides a greater length of the elastic chain to facilitate disimpaction. ${ }^{22}$ Usually, in case of impaction, PMFM is likely to rotate around the palatal cusp. ${ }^{23}$ Thus, the buccal and palatal extensions were incorporated into the design to allow distal and buccal drift of the impacted molars. ${ }^{22}$ These extensions were adjusted to avoid tissue impingement and were adapted as distally as possible to accommodate the full width of the permanent molar. ${ }^{22}$ The crossarch anchorage preserved the leeway space and prevented the rotation and forward movement tendency of PFM. ${ }^{4-8}$ Once the appliance was cemented, the chain elastics were placed using Mathieu orthodontic pliers.

After cementation, the patient returned every two weeks for oral hygiene control, monitoring the distal tipping of PMFMs, and activation of the appliance by shortening the elastics, thus increasing the disimpaction force. ${ }^{7-14,22}$

After six weeks of treatment, a routine examination revealed that PMFMs were freed from their ectopic position. Periapical radiographs confirmed the latter observation. The chain elastics were then removed, but the appliance was kept in place allowing the passive eruption of the molars. Two weeks later, the correction was complete and the appliance was removed. ${ }^{22}$

Kurol and Bjerklin stated that the primary second molars are likely to persist as natural space maintainers until the normal shedding time. ${ }^{24}$ It can be explained by the correction of the eruption path of PMFM and the deposition of the secondary dentin obliterating the exposed area of resorption. ${ }^{4-9}$ This can be applicable to our case, since 55 and 65 initially presented grade III resorption and remained without any further damage or mobility at the end of the treatment.

A routine follow-up was scheduled every six months to monitor the establishment of the occlusion as the patient was transitioned to the permanent dentition. ${ }^{8,9}$

Compared to other techniques, this appliance requires an extended chairside and laboratory time. ${ }^{9}$ It can be time consuming, as frequent changing of elastics is needed. ${ }^{9}$ Due to the inclination of the impacted teeth, achieving a good isolation for the bonding of the occlusal buttons can be a challenge for clinicians. ${ }^{14}$ Despite these disadvantages, the modified Croll's appliance has many positive aspects. Indeed, it respects the integrity of the epithelial attachment, especially in the case of teeth with tight contacts where it is not possible to place separators. ${ }^{9}$ It is pain-free and requires a short treatment time. ${ }^{7}$ Since the appliance used in this case is fixed, minimal patient compliance was needed for the success of the treatment. ${ }^{8}$

\section{Conclusion}

Nowadays, many interceptive orthodontic treatments for the correction of EE are available. Clinicians should establish a proper diagnosis and choose the best appliance that will satisfy not only the clinical aspect of their treatment, but also the patient's well-being.
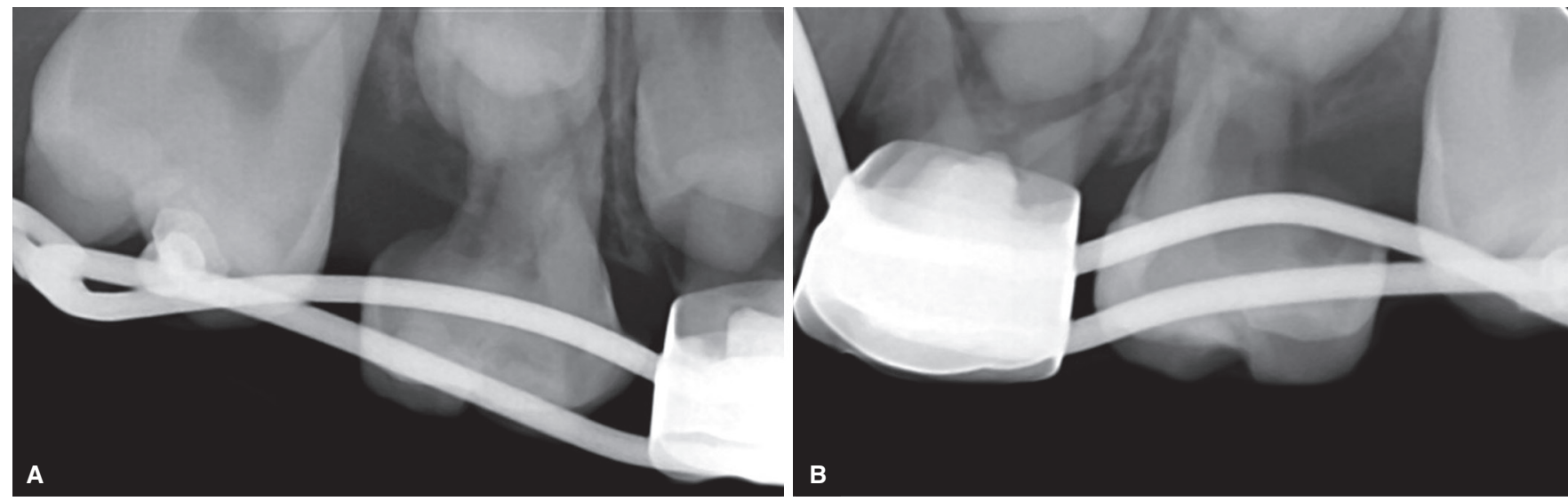

Figs 5A and B: Postoperative radiographs showing the uprighted permanent molars (16) and (26) 


\section{Clinical Significance}

This report shows that if the ectopic eruption is detected earlier, then more pediatric dentists have a chance to minimize the difficulty of future orthodontic treatment and limit the extent of malocclusion to the permanent dentition.

\section{References}

1. Chapman H. First upper permanent molar partially impacted against second deciduous molar. Int J Orthod Oral Surg Radiogr 1923;9: 339-345. DOI: 10.1016/S0099-6963(23)80023-0.

2. American Academy of Pediatric Dentistry. Guideline on Management of the Developing Dentition and Occlusion in Pediatric Dentistry. Pediatr Dent 2016 Oct;37(6):289-301.

3. $\mathrm{Nam} \mathrm{OH}, \mathrm{Ahn} \mathrm{HJ}$, et al. Treatment of ectopic permanent maxillary first molar using a K-loop. J Clin Pediatr Dent 2015;39(4):387-391. DOI: 10.17796/1053-4628-39.4.387.

4. Nagaveni NB, Radhika NB. Interceptive orthodontic correction of ectopically erupting permanent maxillary flrst molar. A case report. Virtual Journal of Orthodontics December 2010.

5. Young DH. Ectopic eruption of the first permanent molar. J Dent Child 1957;24:153-162.

6. Poornima P, Pathak $S$, et al. Simple treatment of ectopic eruption with a stainless steel crown. CHRISMED J Health Res 2015;2:289-291. DOI: 10.4103/2348-3334.158719.

7. Pomarico L, Guimarães $L$, et al. Ectopic eruption of the maxillary central permanent incisors and mandibular first permanent molars: report of an unusual case. Quintessence Int 2006;37:677-683.

8. Machado AW, Braga E. Unilateral impaction of a maxillary first molar treated in the transitional dentition. Pediatr Dent 2014;36: 502-504.

9. Badakar C, Kathariya M, et al. Management of bilateral ectopically erupting mandibular molars using Humprey's appliance - a case report. IJRID 2014;4(1):58-64.

10. Pulver F. The etiology and prevalence of ectopic eruption of the maxillary first permanent molar. ASDC J Dent Child 1968;35(2): 138-146.
11. Guzeler I, Sara S, et al. Primary tooth radicular resorption as a consequence of self-corrected ectopic eruption: 2 unusual cases. J Dent Child 2011;78(3):159-162.

12. Kupietzky A. Correction of ectopic eruption of permanent molars utilizing the brass wire technique. Pediatr Dent 2000;22:408-412.

13. Barberia-Leache E, Suarez-Clúa MC, et al. Ectopic eruption of the maxillary first permanent molar: Characteristics and occurrence in growing children. Angle Orthod 2005;75:610-615. DOI: 10.1043/0003-3219(2005)75[610:EEOTMF]2.0.CO;2.

14. Weinberger SJ. Correction of bilateral ectopic eruption of first permanent molars using a fixed appliance. Pediatr Dent 1992;14(6):382-383.

15. Mitsuhata $C$, Konishi $Y$, et al. Treatment of ectopic eruption of permanent mandibular first molars with innovative dental appliances. Eur J Paediatr Dent 2014;15(2 Suppl):181-183.

16. Witchel J. A Simple Treatment for Ectopic Eruption. Ontario Dentist January/February 2015;42-44.

17. Barberia-Leache E, Suarez-Clua M, et al. Ectopic Eruption of the Maxillary First Permanent Molar: Characteristics and Occurrence in Growing Children. Angle Orthod 2005;75(4):610-615. DOI: 10.1043/0003-3219(2005)75[610:EEOTMF]2.0.CO;2.

18. Roberts MW. Treatment of ectopically erupting maxillary permanent first molars with a distal extended stainless steel crown. J Dent Child 1986;53:430-432.

19. Hardwick F. What is ectopic eruption of the maxillary molars, and how should it be managed? JACD 2007;73(6):491-492

20. Nolla CM. The development of permanent teeth. J Dent Child 1960;27:254-256.

21. Croll TP. Correction of first permanent molar ectopic eruption. Quintessence Int 1984;15(12):1239-1246.

22. Kennedy DB. Clinical tips for the Halterman appliance. Pediatr Dent 2007;29:327-329.

23. Ackerman JL, Proffit WR. Diagnosis and planning treatment. Graber TM, Swain BF. Current Orthodontic Concepts and Techniques. Philadelphia, Pa: WB Saunders Co, 1975; p. 51.

24. Kurol J, Bjerklin K. Resorption of maxillary second primary molars caused by ectopic eruption of the maxillary first permanent molar. A longitudinal and histological study. ASDC J Dent Child 1982;49: 273-279. 